\title{
MOF(ZM)/Potassium Citrate-Derived Composite Porous Carbon and Its Electrochemical Properties
}

\author{
Guoqing Zhang1, Pitao Wang2,3, Chi Zhang2,3, Lili Wu',3, Jing Yang2,3, Mingjian Zhang2,3, \\ Zhong Ren ${ }^{2,3}$, Jianqiang Zhang, ${ }^{2,3}$, Heming Luo ${ }^{2,3^{*}}$
}

${ }^{1}$ School of Material Science and Engineering, Lanzhou University of Technology, Lanzhou, China

${ }^{2}$ Key Laboratory of Low Carbon Energy and Chemical Engineering of Gansu Province, Lanzhou, China

${ }^{3}$ School of Petrochemical Technology, Lanzhou University of Technology, Lanzhou, China

Email: *luohm666@163.com

How to cite this paper: Zhang, G.Q., Wang, P.T., Zhang, C., Wu, L.L., Yang, J., Zhang, M.J., Ren, Z., Zhang, J.Q. and Luo, H.M. (2021) MOF(ZM)/Potassium CitrateDerived Composite Porous Carbon and Its Electrochemical Properties. Journal of Minerals and Materials Characterization and Engineering, 9, 462-479.

https://doi.org/10.4236/jmmce.2021.95031

Received: July 23, 2021

Accepted: August 29, 2021

Published: September 1, 2021

Copyright $\odot 2021$ by author(s) and Scientific Research Publishing Inc. This work is licensed under the Creative Commons Attribution International License (CC BY 4.0).

http://creativecommons.org/licenses/by/4.0/ (c) (i) Open Access

\begin{abstract}
Metal-organic frameworks are compounds with a reticulated skeletal structure formed by chemically bonding inorganic and organic units that are widely used in many fields, such as photocatalysis, gas separation and energy storage, because of their unique structures. In this paper, we prepared a metal-organic framework $\left[\left(\mu_{2} \text {-2-methylimidazolyl }\right)_{12}-\mathrm{Zn}(\mathrm{ii})_{6}-\mathrm{H}_{18} \mathrm{O}_{10}\right]_{\mathrm{n}}(\mathrm{ZM})$ with well-developed pores and high specific surface area of MOFs by the solution method. And MOF-derived porous carbon was prepared by the direct charring method in an argon atmosphere using a mixture of $\mathrm{ZM}, \mathrm{ZM}$ and potassium citrate as carbon precursors. Characterization analysis revealed that the maximum specific surface area of ZMPC-800-1:15 was $2014.97 \mathrm{~m}^{2} \cdot \mathrm{g}^{-1}$, and the pore size structure was mainly mesoporous. At a current density of $1.0 \mathrm{~A} \cdot \mathrm{g}^{-1}$ the specific capacitance of ZMC-800 and ZMPC-800-1:15 was $121.3 \mathrm{~F} \cdot \mathrm{g}^{-1}$ and $226.6 \mathrm{~F} \cdot \mathrm{g}^{-1}$, respectively, with a substantial increase of $86.8 \%$. The specific capacitance of ZMPC-800-1:15 decays to $168.8 \mathrm{~F} \cdot \mathrm{g}^{-1}$, with a decay rate of $25.5 \%$, when the current density increases to $10.0 \mathrm{~A} \cdot \mathrm{g}^{-1}$. After 5000 constant current charge/ discharge cycles, the capacitance retention rate was still $96.41 \%$. These results prove that the application of MOF-derived carbon materials in future supercapacitors is very promising.
\end{abstract}

\section{Keywords}

Metal-Organic Framework, Room Temperature Solution Method, Derived Composite Porous Carbon, Electrochemical Performance, Supercapacitor 


\section{Introduction}

With the continuous development of supercapacitor electrode materials, composite materials, metal oxides, functional carbon materials and conducting polymers have been successfully applied to supercapacitors [1] [2]. Among them, carbon materials have abundant pore structures, excellent electron transport and stable chemical properties [3]. The excellent property of carbon materials is determined by the structure of the carbon source, charring temperature and charring time [4] [5]. The high specific surface area and well-developed pore structure of conventional carbon materials comply with the requirements of bilayer structures for charge transfer and high specific capacitance, but there are certain deficiencies in pore size regulation and specific surface area utilization [6].

MOF materials are crystalline framework structures synthesized by assembling metal clusters/ion-organic ligands that have the advantages of high porosity and a large specific surface area, but the stability of their frameworks is generally poor, and the framework structure is prone to collapse during high-temperature carbonization, resulting in the degradation of the specific surface and porosity as well as the electrochemical properties of the derived porous carbon, which affects the application of carbon materials derived from MOFs in supercapacitors [7]. Therefore, it remains a popular research topic to study and development of high energy storage porous carbon derived from MOFs [8] [9] [10].

Hong, S. et al. [11] synthesized layered porous carbons (HPCs) by direct charring zinc-based MOFs, which have a specific surface area of $1000-1820 \mathrm{~m}^{2} \cdot \mathrm{g}^{-1}$ and are microporous, mesoporous and macroporous. At a scan rate of 10 $\mathrm{mV} \cdot \mathrm{s}^{-1}$, the specific capacitance of HPCs is $164-203 \mathrm{~F} \cdot \mathrm{g}^{-1}$. Layered porosity and nitrogen doping in HPCs were shown to improve their conductivity and specific capacitance. Zhong, S. et al. [12] prepared multilayer porous carbon by calcining Metal-Organic Framework (MOF)/Chitosan (CS) composites and discussed the effect of chitosan additives on the pore-forming structure. The specific surface area of NPC-2 is $2375 \mathrm{~m}^{2} \cdot \mathrm{g}^{-1}$, and the pore volume is $2.49 \mathrm{~cm}^{3} \cdot \mathrm{g}^{-1}$. The degree of graphitization for NPC-2 is higher than that of NPC without chitosan. The excellent properties of NPC-2 facilitate rapid ion diffusion and transport. At a current density of $0.05 \mathrm{~A} \cdot \mathrm{g}^{-1}$, NPC-2 has a high specific capacitance of $199.9 \mathrm{~F} \cdot \mathrm{g}^{-1}$ and good multiplicative performance in an electrolyte of $1 \mathrm{M} \mathrm{H}_{2} \mathrm{SO}_{4}$. At a current density of $2 \mathrm{~A} \cdot \mathrm{g}^{-1}$, the capacitance loss of NPC-2 was $21 \%$ after 10,000 cycles, showing excellent cycling stability of MOF-derived composite porous carbon NPC-2.

In our study, a metal-organic framework material $\left[\left(\mu_{2} \text {-2-methylimidazolyl }\right)_{12}-\mathrm{Zn}(\mathrm{ii})_{6}-\mathrm{H}_{18} \mathrm{O}_{10}\right]_{\mathrm{n}}$ was synthesized by the solution method to prepare porous carbon derived from a high specific surface area and high porosity by direct carbonization of MOFs and carbonization of MOFs and a potassium citrate mixture, respectively. The energy storage performance of the MOF-derived porous carbon materials can be enhanced by the effect of carbonization-selfactivation of potassium citrate. This study provides experimental data on the 
preparation of high-performance MOF-derived composite porous carbon materials.

\section{Experimental}

\subsection{Material Preparation}

\subsubsection{Preparation of Metal-Organic Framework}

$\left[\left(\mu_{2} \text {-2-Methylimidazolyl }\right)_{12}-\mathrm{Zn}(\mathrm{ii})_{6}-\mathrm{H}_{18} \mathrm{O}_{10}\right]_{\mathrm{n}}$

Metal-organic framework $\left[\left(\mu_{2} \text {-2-methylimidazolyl }\right)_{12}-\mathrm{Zn}(\mathrm{ii})_{6}-\mathrm{H}_{18} \mathrm{O}_{10}\right]_{\mathrm{n}}$ was prepared by the reported method [13]. $1.64 \mathrm{~g}$ zinc nitrate hexahydrate was dissolved in $80 \mathrm{~mL}$ of methano1, stirred magnetically for $10 \mathrm{~min}$, and recorded as solution A. $3.7 \mathrm{~g}$ 2-methylimidazolyl dissolved in $80 \mathrm{~mL}$ of methanol, stirred magnetically for $10 \mathrm{~min}$, and recorded as solution B. Solution A was mixed into solution B dropwise with stirring and observed to form a milky solution, stirred at high speed at room temperature for $24 \mathrm{~h}$, centrifuged, washed with methanol several times. Then the products were dried in a vacuum oven at $60{ }^{\circ} \mathrm{C}$ for $12 \mathrm{~h}$ to get a white solid powder which is $\left[\left(\mu_{2}-2 \text {-methylimidazolyl }\right)_{12}-\mathrm{Zn}(\mathrm{ii})_{6}-\mathrm{H}_{18} \mathrm{O}_{10}\right]_{n}$, abbreviated as ZM.

\subsubsection{Preparation of ZM-Derived Porous Carbon ZMC-T}

The $\mathrm{ZM}$ was weighed into a square porcelain boat, transferred to a tube furnace. Then, we increased it to the desired temperature $\left(600^{\circ} \mathrm{C}, 700^{\circ} \mathrm{C}, 800^{\circ} \mathrm{C}\right.$, and $\left.900^{\circ} \mathrm{C}\right)$ for $2 \mathrm{~h}$ with the heating rate of $5^{\circ} \mathrm{C} \cdot \mathrm{min}^{-1}$ under an argon atmosphere. After natural cooling, the products were collected, washed with $1 \mathrm{M}$ hydrochloric acid and deionized water. Finally, the porous carbon ZMC-T was obtained after drying at $60^{\circ} \mathrm{C}$ for $12 \mathrm{~h}$, where $\mathrm{C}$ stands for porous carbon, and $\mathrm{T}$ stands for carbonization temperature. The charring temperature is determined by the thermogravimetric (TG) curve of sample ZM, the charring time and the heating rate were determined according our previous work [14] [15].

\subsubsection{Preparation of ZM-Derived Composite Porous Carbon ZMPC-T-A} The ZM powder and potassium citrate were weighed according to different mass ratios $(1: 5,1: 15,1: 25)$ and placed in a mortar and fully ground for $10 \mathrm{~min}$. Then the mixture transferred to a tube furnace, warmed to different temperatures $\left(600^{\circ} \mathrm{C}, 700^{\circ} \mathrm{C}, 800^{\circ} \mathrm{C}, 900^{\circ} \mathrm{C}\right)$ for $2 \mathrm{~h}$ with the heating rate of $5^{\circ} \mathrm{C} \mathrm{min}{ }^{-1}$ under an argon atmosphere. After natural cooling, the black powder obtained was washed with $\mathrm{HCl}(1 \mathrm{M})$ and $\mathrm{DI}$ water three times, thoroughly dried at $60^{\circ} \mathrm{C}$ overnight and then labeled as ZMPC-T-A, where $\mathrm{P}$ represents potassium citrate, $\mathrm{C}$ represents porous carbon, $\mathrm{T}$ represents carbonization temperature, and $\mathrm{A}$ represents the mass ratio of ZM powder to potassium citrate.

\subsection{Structural Characterization}

$\mathrm{X}$-ray powder diffraction (XRD) patterns of $\mathrm{MOF}$ and porous carbon were collected on an X-ray diffractometer (Panaco, The Netherlands) with $\mathrm{Cu}$ Ka radiation over the $2 \theta$ range of $5^{\circ}-90^{\circ}$. According to standard SN/T 3078.2-2015, a thermogravimetric analyzer (Hi-Res TGA 2950, TA, USA) was used to charac- 
terize the thermal stability of the MOF materials under the following test conditions: a nitrogen atmosphere and $30^{\circ} \mathrm{C}-900^{\circ} \mathrm{C}$. Measurements were made using a Raman spectrometer (JY-HR800, Horiba, Japan) in an excitation wavelength of $532 \mathrm{~nm}$ at room temperature. Imaging of the porous carbon materials was performed using an S-4800 field emission Scanning Electron Microscope (SEM) and a JEOL JEM-2010 high-resolution Transmission Electron Microscope (TEM). According to the GB/T19587-2017/ISO 9277:2010, the specific surface areas, the pore volume and pore size distribution were measured using ASAP 2020 (Mack Instruments, USA). X-ray Photoelectron Spectroscopy (XPS) was used to characterize the surface elemental composition of materials on an Escalab 250Xi spectrometer (Thermo Scientific, USA).

\subsection{Working Electrode Preparation}

ZMPC-T-A, acetylene black and PTFE were weighed according to a mass ratio of 8.5:1:0.5, and the mass was controlled at approximately $5.0 \mathrm{mg}$. A suitable amount of anhydrous ethanol was added to sticky, which was then evenly coated on a nickel foam sheet, dried under vacuum at $80^{\circ} \mathrm{C}$ for $24 \mathrm{~h}$, and cooled and pressed under $15 \mathrm{MPa}$ for $90 \mathrm{~s}$; thus, the working electrode was obtained.

\subsection{Electrochemical Performance Test}

Selecting of suitable supercapacitor electrode materials, a traditional three-electrode system of CHI660E electrochemical workstation was used for the electrochemical measurements. The working electrode was ZMC-T and ZMPC-T-A, the counter electrode was a platinum sheet electrode $\left(1.5 \mathrm{~cm}^{2}\right)$, while the reference electrode was a glycerol electrode. Cyclic Voltammetry (CV), Galvanostatic ChargeDischarge (GCD), and electrochemical impedance spectroscopy (EIS) tests were performed in $6 \mathrm{M} \mathrm{KOH}$ electrolyte at different current densities. The specific capacitance $C\left(\mathrm{~F} \cdot \mathrm{g}^{-1}\right)$ based on the GCD curve was calculated by Equation (1):

$$
C=\frac{I \cdot \Delta t}{m \cdot \Delta V}
$$

where $C$ : specific capacitance (F.g $\left.{ }^{-1}\right) ;$ : discharge current (A); $\Delta t$. discharge time (s); m: mass (g); $\Delta V$ : voltage change ( $V$ ).

The specific capacitance $C\left(\mathrm{~F} \cdot \mathrm{g}^{-1}\right)$ based on the $\mathrm{CV}$ curve can be calculated by Equation (2):

$$
C=\frac{\int i(V)}{2 \cdot v m \Delta V} \mathrm{~d} V
$$

where: $i:$ current as a function of voltage $(V) ; v \cdot$ scan rate $\left(\mathrm{mV} \cdot \mathrm{s}^{-1}\right)$.

\section{Results and Discussion}

\subsection{Physical Phase Analysis}

\subsubsection{XRD and TG-DTA Analysis of ZM}

The X-ray diffraction pattern of sample ZM is shown in Figure 1(a). Strong 


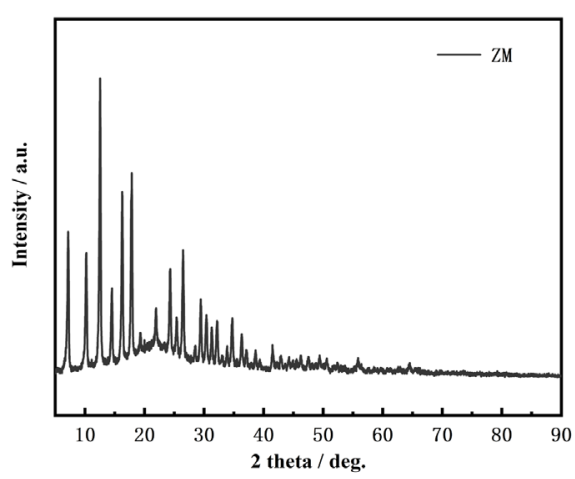

(a)

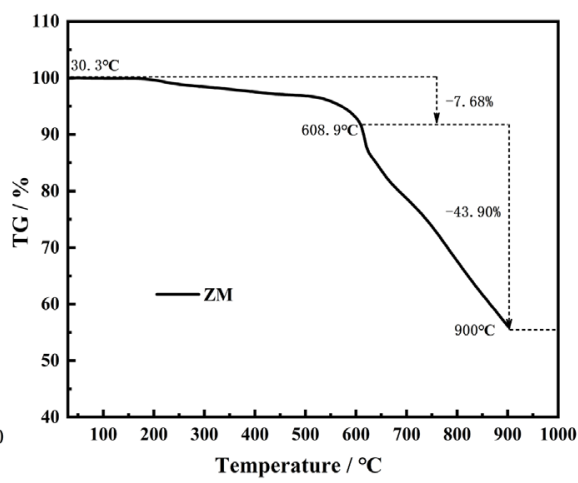

(b)

Figure 1. (a) XRD and (b) TG curve of ZM.

diffraction peaks appear at $2 \theta=7.18^{\circ}, 10.24^{\circ}, 12.54^{\circ}, 14.54^{\circ}, 16.24^{\circ}, 17.84^{\circ}$, $24.32^{\circ}$, and $26.48^{\circ}$, which indicate that the synthesized ZM has high crystallinity, and these peaks are basically consistent with the results in the literature [13]. Figure 1(b) shows the thermogravimetric (TG) curve of sample ZM. The test temperature range is $30^{\circ} \mathrm{C}-900^{\circ} \mathrm{C}$, and the decomposition process is split into two stages. When the temperature was raised from $34.7^{\circ} \mathrm{C}$ to $608.9^{\circ} \mathrm{C}$, the sample shows a gentle weight loss in the process of continuous heat absorption, and a 7.68\% mass loss occurs at this stage. This stage is mainly attributed to the evaporation of solvent molecules and water molecules in the ZM crystals and the resulting gas dissipation, resulting in weight loss. As the temperature increases, the ZM framework gradually collapses and is destroyed at high temperatures, some of it undergoes charring, and the metal ions contained in the material are oxidized to become metal oxides. Between $608.9^{\circ} \mathrm{C}$ and $900^{\circ} \mathrm{C}, 43.9 \%$ mass loss occurs. Charring of the material mainly occurs at this stage, and much stable carbon material is formed. With a further increase in temperature, the charring deepens gradually, the metal zinc atoms contained in ZM are oxidized and then reduced, and the high temperature makes some of them escape by gasification [16] [17] [18].

\subsubsection{XRD Analysis of the Composite Porous Carbon Material ZMPC-T-1:15}

The X-ray diffraction pattern of composite porous carbon ZMPC-T-1:15 was analyzed in the test range of $2 \theta=5^{\circ}-90^{\circ}$, as shown in Figure 2. The composite porous carbon ZMPC-T-1:15 has an obvious characteristic diffraction peak at $2 \theta$ $=21.8^{\circ}$, and the (002) peak is substantially shifted to the left compared with graphite $\left(2 \theta=26.4^{\circ}\right)$, indicating that the (002) interplanar layer spacing between the planes of the composite porous carbon has been extended, which is due to the mild oxidation of the carbon material during the carbonization process and the successive changes in the morphology of the metal elements in the MOFs at high temperatures, leading to the distortion of the graphite lattice [19] [20]. The (002) diffraction peaks gradually increase in intensity with increasing carbonization temperature, indicating the enhanced structural ordering and graphitization of the carbon material. 


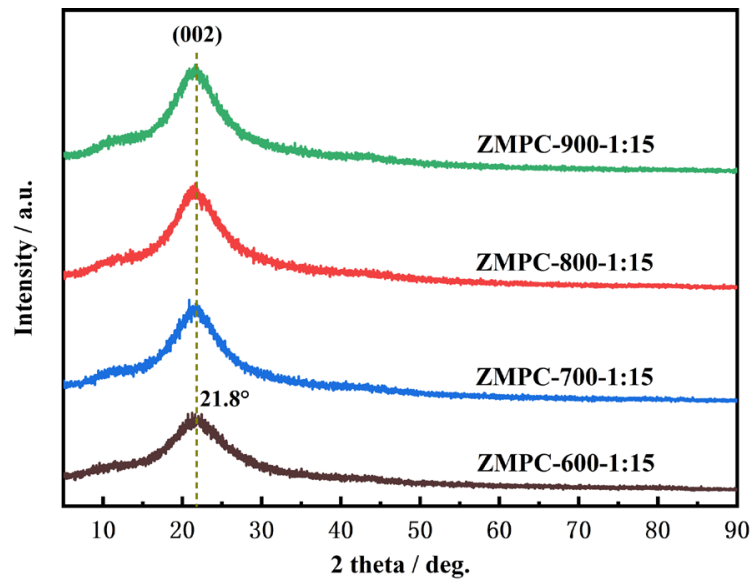

Figure 2. X-ray diffraction pattern of composite porous carbon ZMPC-T-1:15.

\subsubsection{Raman Analysis of Composite Porous Carbon ZMPC-T-1:15}

The intensity ratio of the D-band to the G-band $\left(R=I_{D} / I_{G}\right)$ has become a common scientific research method as a criterion for estimating the defect content on the surface of carbon materials [21]. A decrease in $\mathrm{R}$ is key evidence of the deepening of carbonization [22]. Figure 3 shows the Raman spectrum of the composite porous carbon ZMPC-T-1:15. The graphitization degree of a carbon material can be characterized by an $\mathrm{R}$ value; a higher value of $\mathrm{R}$ reflects more defects in the crystal and a lower degree of graphitization. Two obvious diffraction peaks appear at $1365.4 \mathrm{~cm}^{-1}$ in the D-band and $1585.2 \mathrm{~cm}^{-1}$ in the G-band, and the $\mathrm{R}$ values of ZMPC-600-1:15, ZMPC-700-1:15, ZMPC-800-1:15 and ZMPC-900$1: 15$ are calculated to be $1.94,1.44,1.21$, and 2.72 , respectively. The R-value decreases and then increases as the charring temperature increases. This is because during the high-temperature charring process, the MOF and potassium citrate structures are bound to undergo chemical bond breakage and structural collapse, the carbon atoms in the organic ligand fragments formed by the collapse pass through a series of rearrangements [23] [24], and the carbon materials formed by the rearrangements increase in graphitization with increasing charring temperature; thus, the R-value gradually decreases. However, a charring temperature that is too high drives the carbon atoms to rearrange too fast during the charring process, and the defects produced may increase; thus, the R-value gradually increases.

\subsection{Microstructure}

\subsubsection{Microscopic Morphological Analysis}

Microstructural morphology and elemental distribution images of the composite porous carbon ZMPC-800-1:15 are characterized and shown in Figure 4 using TEM and SEM. Figure 4(a) and Figure 4(b) shows SEM images of ZMPC-800-1:15 at different scanning magnifications. The material consists of irregular cubic blocks stacked and connected with each other, forming numerous intricate pore channels at various levels, which give ZMPC-800-1:15 a graded pore structure. Figure 4(c) and Figure 4(d) shows TEM images of the composite porous carbon 


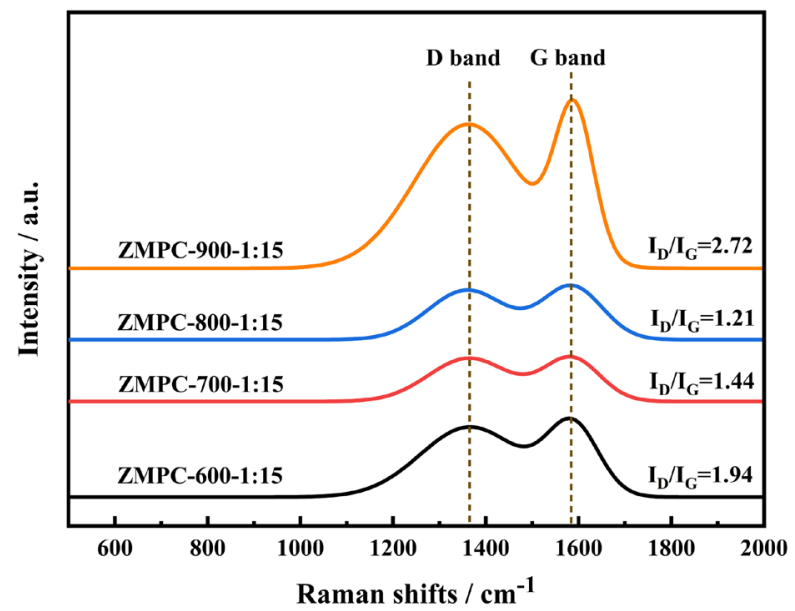

Figure 3. Raman spectra of composite porous carbon ZMPC-T-1:15.

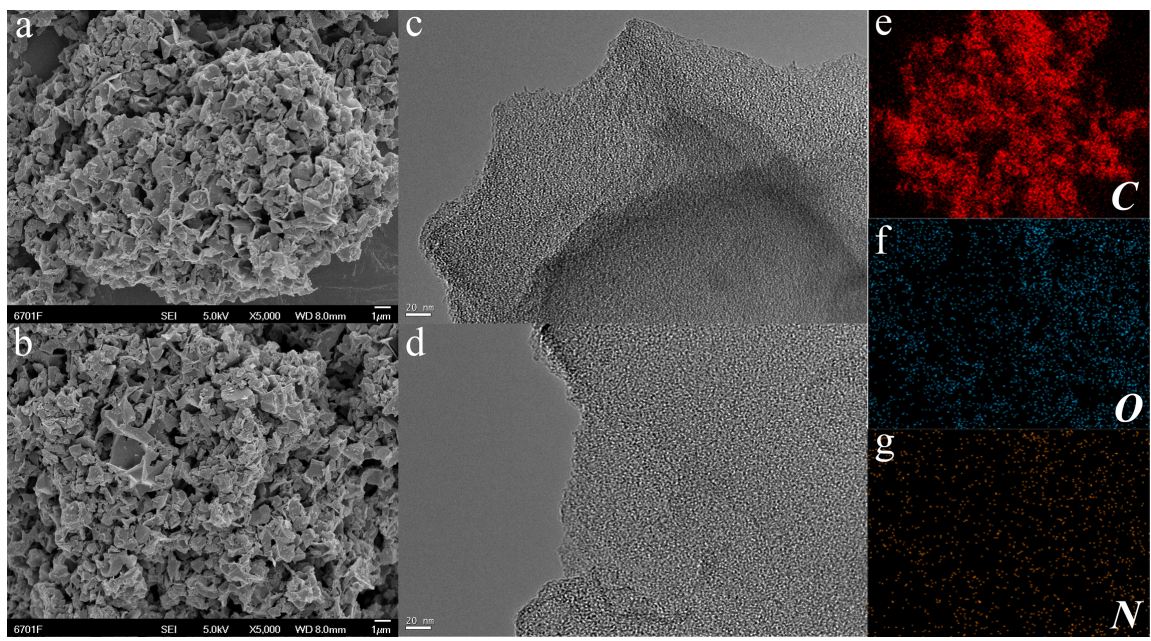

Figure 4. ((a), (b)) SEM images, ((c), (d)) TEM images and ((e)-(g)) elemental distribution images of composite porous carbon ZMPC-800-1:15.

ZMPC-800-1:15. Abundant micromesopores are uniformly distributed in different parts of the sample, which indicates that the carbon material has well-developed pore channels and high porosity, and the edges of the carbon material show a tendency of thin lamellar graphene, showing that the porous carbon ZMPC-800-1:15 has a developed pore structure and excellent electrical conductivity. Porous carbon ZMPC-800-1:15 has a developed void structure and excellent electrical conductivity. It indicated that potassium citrate can provide a part carbon for the composite porous carbon material, and potassium element is helpful to develop pore structure for the composite porous carbon material, which make the composite porous carbon have a large specific surface area. Figures $4(\mathrm{e})-(\mathrm{g})$ shows the elemental distribution of the composite porous carbon ZMPC-800-1:15 in terms of carbon, oxygen and nitrogen. The elemental distribution of oxygen is relatively small and evenly distributed, and the trace nitrogen is scattered, indicating that the composite porous carbon ZMPC-800-1:15 is a carbon material formed by abundant carbon as the skeleton, bonded with a small amount of 
oxygen and trace nitrogen. The existence of nitrogen and oxygen elements in the carbon material can enhance the wettability of the material with the electrolyte and enhance its electrochemical performance.

\subsection{2. $\mathrm{N}_{2}$ Adsorption/Desorption}

The use of gas adsorption/desorption to characterize the structure of porous solids and fine powders is an effective tool for scientific research, and a novel approach based on density flooding theory and molecular simulation allows an accurate and comprehensive analysis of the pore structure [25]. Figure 5(a) shows the nitrogen adsorption/desorption curves of porous carbon ZMC-800. The nitrogen adsorption-desorption isotherms and the corresponding pore-size distribution curves of porous carbon ZMC-800 are showed in Figure 5(a) and Figure 5(b). At different carbonization temperatures, the nitrogen adsorption-desorption isotherms and the pore size distribution curves of porous carbon ZMPC-T-1:15 are showed in Figure 5(c) and Figure 5(d). As shown in Figure 5(c), the composite porous carbon has similar curve shapes at different carbonization temperatures, and the adsorption/desorption curve type is typical Type IV I and IV according to the six classifications of physical adsorption isotherms by IUPAC. In Figure 5(c), a steep section of absorption occurs when the relative pressure $\left(\mathrm{P} / \mathrm{P}_{0}\right)$ is very low $(<0.01)$, which is due to the rapid filling of micropores by nitrogen, thus indicating the presence of abundant micropore structures in the composite porous carbon ZMPC-T-1:15, which displays a hysteresis loop

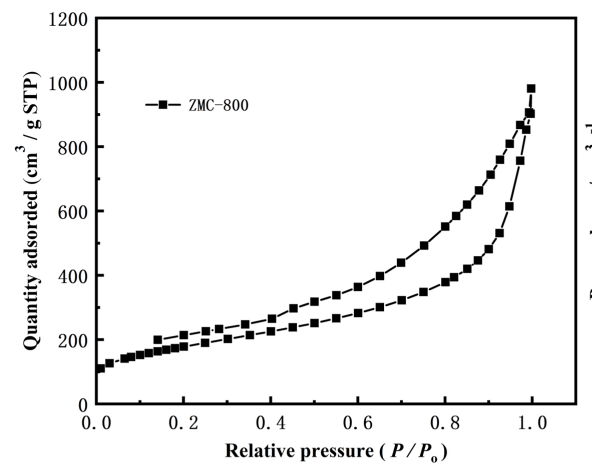

(a)

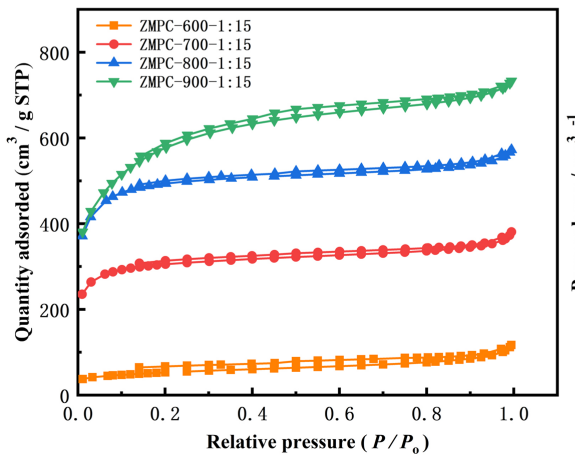

(c)

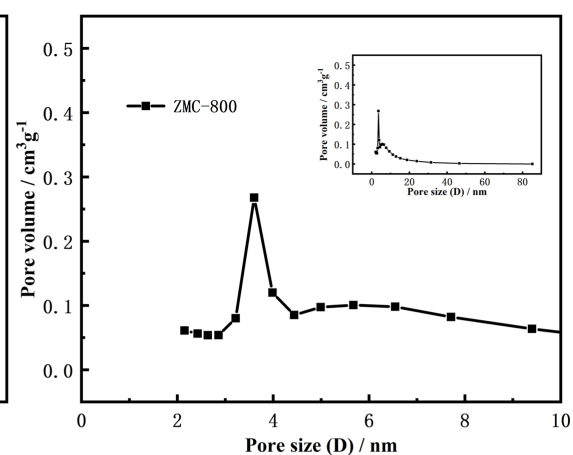

(b)

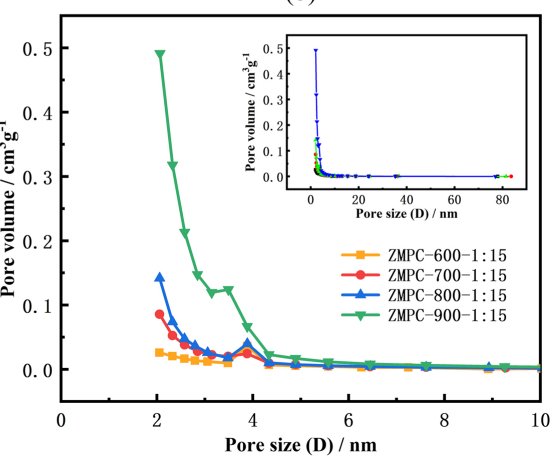

(d)

Figure 5. ((a), (c)) $\mathrm{N}_{2}$ adsorption/desorption curve and ((b), (d)) pore size distribution curve of porous carbon ZMC-800 and composite porous carbon ZMPC-T-1:15. 
of type $\mathrm{H} 2$. This adsorption behavior is mainly determined by the adsorption interactions and intermolecular interactions in the condensed state, and the adsorption curve displays a hysteresis loop of type $\mathrm{H} 2$, so the mesoporous structure of the composite porous carbon material can be inferred. When the relative pressure is near 1, the rise of the curve tends to slow down, indicating that ZMPC-T1:15 has a small amount of macroporous structure. In summary, the composite porous carbon ZMPC-T-1:15 is a graded pore material composed mainly of microporous and mesoporous pores, and the graded pore structure provides the basis for the material to show excellent electrochemical properties. As seen from Figure 5(d), the pore size distribution is mainly concentrated in the mesopores at approximately $3-4 \mathrm{~nm}$, while a few large pores also exist, and the pore size distribution analysis is corresponded with the analysis results of the adsorption/desorption curves.

The specific surface area and pore characteristics data for porous carbon ZMC-800 and composite porous carbon ZMPC-T-1:15 are showed in Table 1. Table 1 shows the specific surface area and pore characteristics data of porous carbon ZMC-800 and composite porous carbon ZMPC-T-1:15. From Table 1, it can be seen that ZMC-800 has a specific surface area of $641.25 \mathrm{~m}^{2} \cdot \mathrm{g}^{-1}$. The specific surface area of the material is increased effectively by adding potassium acid at the same carbonization temperature. The specific surface areas of composite porous carbon ZMPC-600-1:15, ZMPC-700-1:15, ZMPC-800-1:15 and ZMPC900-1:15 were $185.65,1038.24,2014.97$ and $1681.20 \mathrm{~m}^{2} \cdot \mathrm{g}^{-1}$, respectively, and the specific surface areas of the material showed a trend of increasing and then decreasing. Notably, the specific surface area of ZMPC-600-1:15 is only 185.65 $\mathrm{m}^{2} \cdot \mathrm{g}^{-1}$, which may be due to the following reasons: the carbon chain of ZM is broken at a lower carbonization temperature, the rearrangement of carbon atoms in the carbon chain is not complete, the pore structure of ZM-derived carbon material is not well developed, the $\mathrm{K}_{2} \mathrm{O}$ generated by potassium citrate is not enough to induce carbon skeleton etching and pores, and the extension of the carbon skeleton produces thick layers of carbon material, decreasing the specific surface area. After direct carbonization of potassium citrate, the specific

Table 1. Specific surface area and parameter characteristics of porous carbon ZMC-800 and composite porous carbon ZMPC-T-1:15.

\begin{tabular}{cccccccc}
\hline Sample & $\begin{array}{c}S_{\mathrm{BET}} \\
\left(\mathrm{m}^{2} \cdot \mathrm{g}^{-1}\right)\end{array}$ & $\begin{array}{c}S_{\text {mic }} \\
\left(\mathrm{m}^{2} \cdot \mathrm{g}^{-1}\right)\end{array}$ & $\begin{array}{c}S_{\text {mes \& mac }} \\
\left(\mathrm{m}^{2} \cdot \mathrm{g}^{-1}\right)\end{array}$ & $\begin{array}{c}V_{\text {total }} \\
\left(\mathrm{cm}^{3} \cdot \mathrm{g}^{-1}\right)\end{array}$ & $\begin{array}{c}V_{\text {mic }} \\
\left(\mathrm{cm}^{3} \cdot \mathrm{g}^{-1}\right)\end{array}$ & $\begin{array}{c}V_{\text {mes } \& \text { mac }} \\
\left(\mathrm{cm}^{3} \cdot \mathrm{g}^{-1}\right)\end{array}$ & $\begin{array}{c}D_{\text {average }} \\
(\mathrm{nm})\end{array}$ \\
\hline ZMC-800 & 641.25 & 62.34 & 578.91 & 0.69 & 0.02 & 0.67 & 7.30 \\
ZMPC-600-1:15 & 185.65 & 63.65 & 122.00 & 0.16 & 0.03 & 0.13 & 3.35 \\
ZMPC-700-1:15 & 1038.24 & 760.90 & 277.34 & 0.56 & 0.35 & 0.21 & 2.15 \\
ZMPC-800-1:15 & 2014.97 & 802.50 & 1212.47 & 1.11 & 0.35 & 0.76 & 2.17 \\
ZMPC-900-1:15 & 1681.20 & 1226.18 & 455.02 & 0.86 & 0.56 & 0.30 & 2.04 \\
\hline
\end{tabular}

$S_{\text {mes \& mac }}$ : total surface area of mesopores and macropores, $S_{\text {mic }}$ : micropore surface area, $V_{\text {mes \& mac: }}$ total pore volume of mesopores and macropores, $V_{\text {mic }}$ : micropore pore volume. 
surface area of the carbon material is $1940 \mathrm{~m}^{2} \cdot \mathrm{g}^{-1}$ [26], which is slighter lower than that ZMPC-800-1:15. It indicates that ZMPC-800-1:15 has more active site with electrolyte ions to participate in the adsorption and desorption process, which improve the electrochemical performance of the composite porous carbon material.

At a higher carbonization temperature, $\mathrm{ZM}$ and potassium citrate simultaneously undergo the carbonization-self-activation process; that is, ZM and potassium citrate can carbonize at high temperature to form porous carbon materials, and the gas generated by the decomposition of $\mathrm{ZM}$ and potassium citrate enters between the carbon layers, which dissolves the microstructure of carbon materials to form certain pore channels. ZM and potassium citrate decompose and react to produce metal atoms, which help to form the pore structure of carbon materials. Finally, the composite porous carbon material ZMPC-T-1:15 with well-developed pores was formed [26] [27], and it is obvious that the specific surface area of this composite porous carbon material was substantially higher than the specific surface area of ZMC-800 [28]. At different charring temperatures, the pore volumes of the composite porous carbon ZMPC-T-1:15 were 0.16, $0.56,1.11$, and $0.86 \mathrm{~cm}^{3} \cdot \mathrm{g}^{-1}$, and the average pore diameters were $3.35,2.15,2.17$, and $2.04 \mathrm{~nm}$, respectively. Thus, it can be concluded that the composite porous carbon with high specific surface area and suitable pore size can provide the basis for the fast ion and electron transport of the electrolyte and substantially improve the electrochemical specific capacity and multiplicity of the composite porous carbon material.

\subsection{XPS Analysis}

$\mathrm{X}$-ray Photoelectron Spectroscopy (XPS) is an essential tool used to reveal the surface properties of substances, and it can investigate the surface elements, their chemical state and the number of elements based on the binding energy and intensity of the photoelectron peaks [29] [30]. As a surface analysis technique, XPS is useful in scientific research for analyzing the surface chemical state of carbon materials with spectra of $\mathrm{C} 1 \mathrm{~s}$, O1s, etc. By fitting the binding energy values of various types of characteristic peaks compared with the known bonding energies, a detailed understanding of the various bonding types can be obtained [31]. Figure 6 shows the XPS spectrum of the composite porous carbon ZMPC-800-1:15, and Table 2 shows the XPS analysis data of the composite porous carbon ZMPC800-1:15. Figure 6(a) shows that the characteristic peaks of C1s and O1s are located at $284.4 \mathrm{eV}$ and $531.9 \mathrm{eV}$, respectively. Among them, the peak of $\mathrm{C} 1 \mathrm{~s}$ is obvious, and O1s presents only a weak peak. Figure 6(b) shows that the proportions of $\mathrm{C} 1 \mathrm{~s}$ and $\mathrm{O} 1 \mathrm{~s}$ in both are $95.12 \%$ and $4.78 \%$, respectively, with a large presence of carbon. Figure $6(\mathrm{c})$ shows the three types of peaks fitted by $\mathrm{C} 1 \mathrm{~s}$ [32]: $\mathrm{C}=\mathrm{C}$ at $284.4 \mathrm{eV}$ (71.57 at \%), C-C at $285.1 \mathrm{eV}(17.02 \%$ at \%), and $\mathrm{C}-\mathrm{O}$ at $286.1 \mathrm{eV}$ (11.40 at \%). Figure 6(d) shows the three types of peaks fitted by O1s [33], which are $\mathrm{C}=\mathrm{O}$ (ester) bonds at $531.6 \mathrm{eV}, \mathrm{C}=\mathrm{O}$ (carboxyl) bonds at 532.6 $\mathrm{eV}$, and $\mathrm{O}-\mathrm{C}=\mathrm{O}$ bonds at $533.7 \mathrm{eV}$, with percentages of $40.36 \%, 32.87 \%$, and 


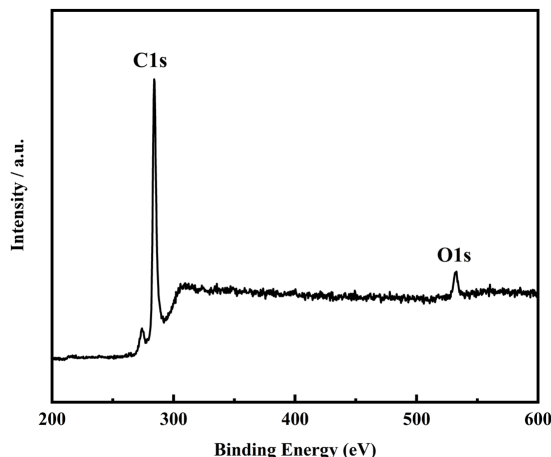

(a)

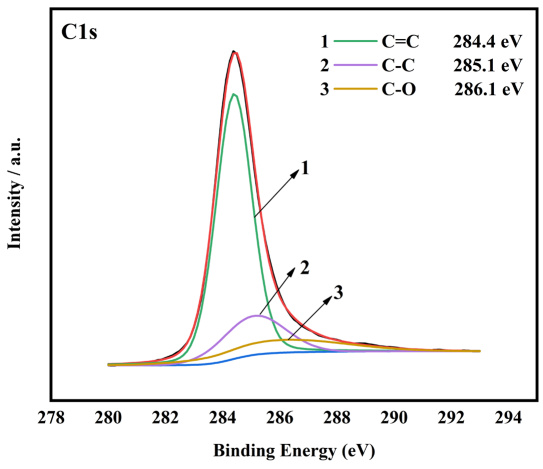

(c)

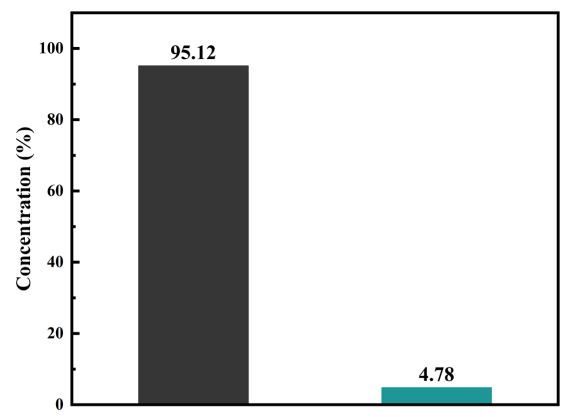

01s

(b)

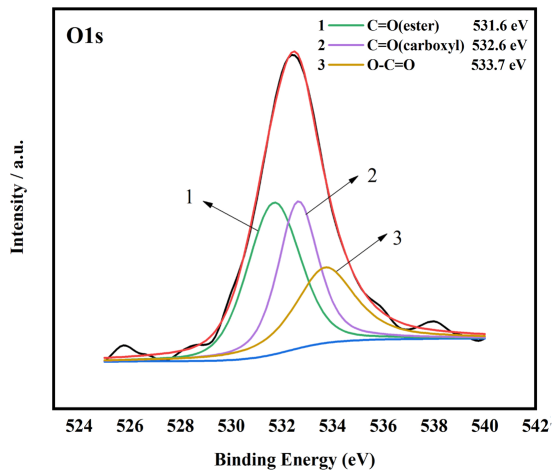

(d)

Figure 6. XPS spectrum of composite porous carbon ZMPC-800-1:15: (a) XPS full spectrum; (b) Content; (c) C1s; (d) O1s.

Table 2. XPS analysis data of composite porous carbon ZMPC-800-1:15.

\begin{tabular}{ccc}
\hline Element & Binding Energy (eV) & Content (at \%) \\
\hline \multirow{2}{*}{ C1s } & 284.4 & 71.57 \\
& 285.1 & 17.02 \\
& 286.1 & 11.40 \\
\hline \multirow{2}{*}{ O1s } & 531.6 & 40.36 \\
& 532.6 & 32.87 \\
& 533.7 & 26.77 \\
\hline
\end{tabular}

$26.77 \%$, respectively. A brief explanation for not seeing the $\mathrm{N}$ element echoes the TEM discussion.

\subsection{Electrochemical Properties}

\subsubsection{Electrochemical Properties of Porous Carbon ZMC-800 and Composite Porous Carbon ZMPC-800-A}

The electrochemical performance of porous carbon ZMPC-800 and composite porous carbon ZMPC-800-A is showed in Figure 7. As shown in Figure 7(a), the GCD curves of porous carbon all show isosceles triangle-like shapes, and the perfect symmetry of the GCD curves indicates that the carbon materials have good charge-discharge performance. At different current densities, the decay 


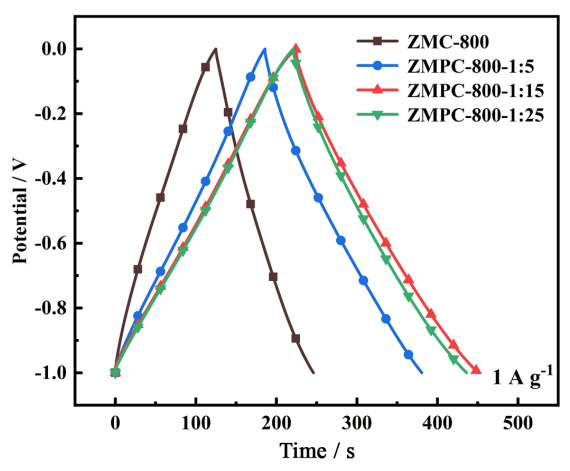

(a)

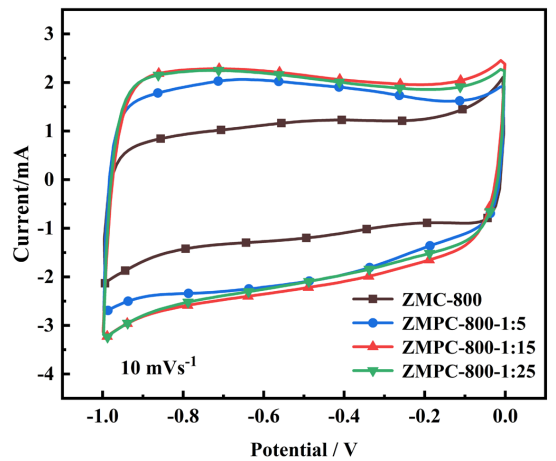

(c)

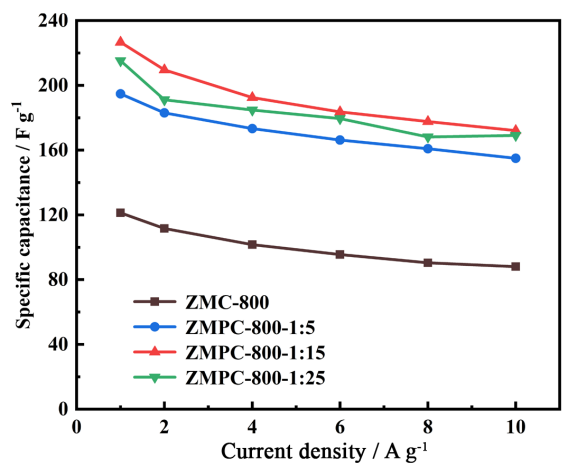

(b)

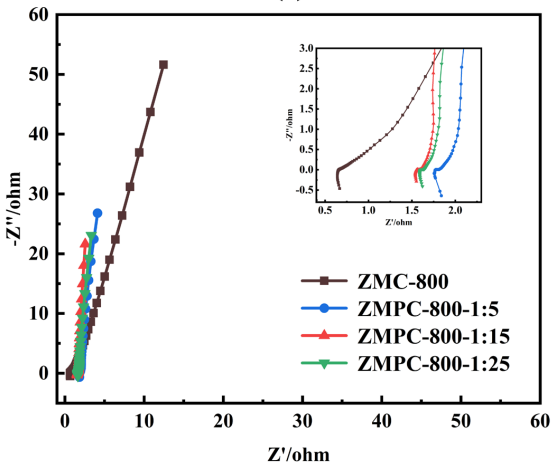

(d)

Figure 7. Electrochemical performance test of porous carbon ZMPC-800 and composite porous carbon ZMPC-800-A: (a) GCD curve at $1.0 \mathrm{~A} \cdot \mathrm{g}^{-1}$; (b) specific capacitance decay curves at different current densities; (c) CV curve at $10 \mathrm{mV} \cdot \mathrm{s}^{-1}$; (d) Nyquist plot.

curves of the specific capacitance of the carbon material are showed in Figure 7(b). At different current densities, the specific capacitance of composite porous carbon ZMC-800-A is substantially higher than that of porous carbon ZMC-800. At the current density of $1.0 \mathrm{~A} \cdot \mathrm{g}^{-1}$, the specific capacitances of porous carbon ZMPC-800 and composite porous carbon ZMPC-800-T are 121.3, 194.8, 226.6, $215.2 \mathrm{~F} \cdot \mathrm{g}^{-1}$, respectively. The capacitance decay of ZMC-800, ZMPC-800-1:5, ZMPC-800-1:15, and ZMPC-800-1:25 were 27.5\%, 20.4\%, 24.0\%, and 21.4\%, respectively, when the current density increased from 1.0 to $10.0 \mathrm{~A} \cdot \mathrm{g}^{-1}$. The capacitance decay rate of composite porous carbon ZMPC-800-A is smaller which indicated the good cycling stability of this carbon material. As shown in Figure 7 (c), at a scan rate of $10 \mathrm{mV} \cdot \mathrm{s}^{-1}$, the $\mathrm{CV}$ curves of porous carbon ZMC-800 and composite porous carbon ZMC-800-A. The porous carbon ZMC-800 and composite porous carbon ZMC-800-A show a certain rectangular shape, which indicate that the porous carbon materials have excellent bilayer capacitance. Among them, ZMPC-800-1:15 has the largest curve integration area, which is consistent with the result that it has the largest specific capacitance. The Nernst chart of porous carbon ZMC-800 and composite porous carbon ZMC-800-A is showed in Figure 7 (d). In the low-frequency region, the Nyquist of all porous carbon materials curve shows a steep straight line, indicating that porous carbon ZMC-800 and composite porous carbon ZMC-800-A have good capacitive behavior. In the high-frequency region, of the arc on the $\mathrm{x}$-axis at high frequency region is called 
an equivalent series resistance (ESR). The date of experience shows that ERS values of porous carbon ZMC-800, and the composite porous carbon ZMPC-800-T $\mathrm{AC}$ are $0.67,1.84,1.55$, and 1.62. Porous carbon ZMC-800 has the smallest resistance, but its lower pore volume and low capacitive characteristics give it obvious limitations. In contrast, since composite porous carbon ZMPC-800-1:15 has the maximum specific surface area and pore volume, the electrolyte ions can enter into the inner space of the porous carbon, and the active sites of the material micropores are the full used, which reveals that composite porous carbon ZMPC-800-1:15 has a higher specific capacitance and a smaller AC impedance resistance.

\subsubsection{Electrochemical Properties of the Composite Porous Carbon Material ZMPC-T-1:15}

The carbonization temperature during carbonization process plays a crucial role in the formation of porous carbon. At a current density of $1.0 \mathrm{~A} \cdot \mathrm{g}^{-1}$, the GCD curves of the composite porous carbon ZMPC-T-1:15 is showed in Figure 8(a). The GCD curves of the composite porous carbon ZMPC-T-1:15 show an isosceles triangle shape at different carbonization temperatures, which demonstrates that the porous carbon ZMPC-T-1:15 has ideal electrochemical properties of a double layer. At a current density of $1.0 \mathrm{~A} \cdot \mathrm{g}^{-1}$, the composite porous carbon ZMPC-T-1:15 have the specific capacitance of 198.9, 206.3, 226.6 and $180.1 \mathrm{~F} \cdot \mathrm{g}^{-1}$,

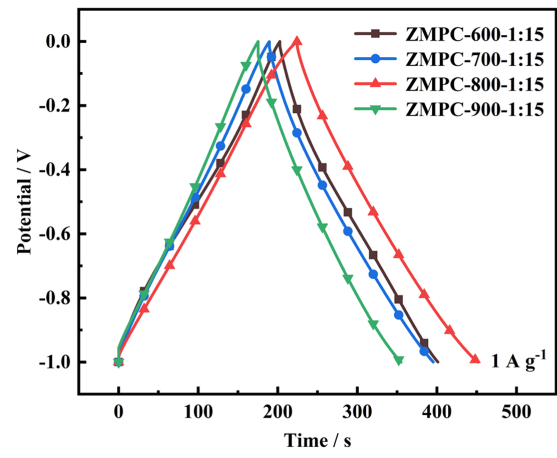

(a)

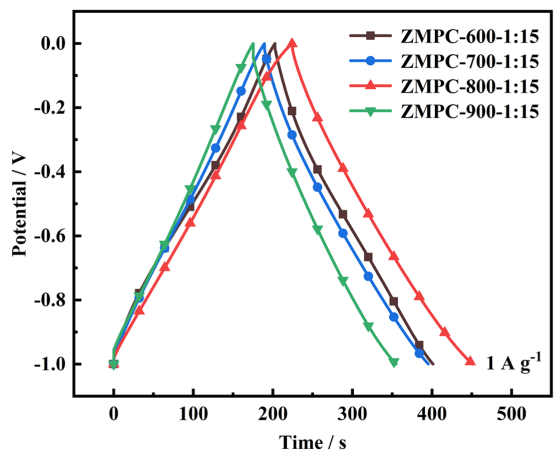

(c)

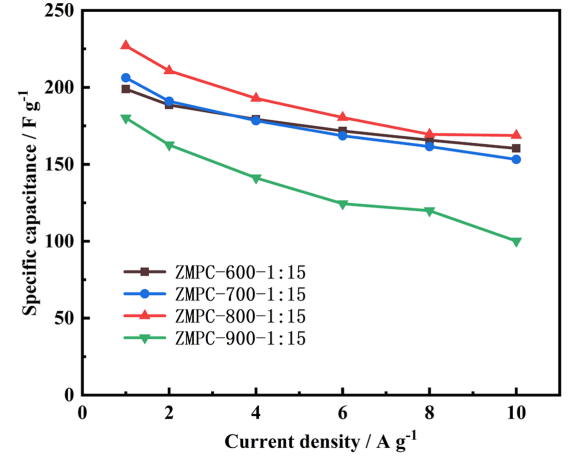

(b)

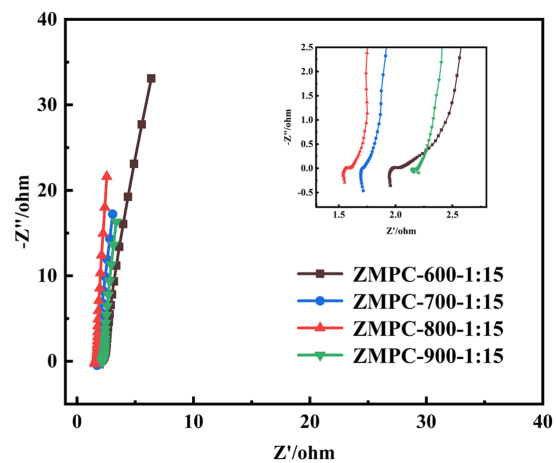

(d)

Figure 8. Electrochemical performance test of composite porous carbon ZMPC-T-1:15: (a) GCD curve at $1.0 \mathrm{~A} \cdot \mathrm{g}^{-1}$; (b) specific capacitance decay curves at different current densities; (c) CV curve at $10 \mathrm{mV} \cdot \mathrm{s}^{-1}$; (d) Nyquist plot. 
respectively, which illustrates a trend of increasing and then decreasing specific capacitance. This result is due mainly to insufficient charring of the ZMPC material at a lower charring temperature and the low porosity of the material, which corresponds to the smaller pore volume and specific surface area measured at a low charring temperature. At carbonization temperatures that were too high, some of the micropores of the composite carbon material ZMPC-T-1:15 collapsed into mesopores, which decreased its pore volume, specific surface area, and charge storage capacity. At different current densities, the decay curves of the specific capacitance of the composite carbon material ZMPC-T-1:15 are showed in Figure 8(b). The specific capacitance decays to $160.4 \mathrm{~F} \cdot \mathrm{g}^{-1}, 153.2 \mathrm{~F} \cdot \mathrm{g}^{-1}, 168.8 \mathrm{~F} \cdot \mathrm{g}^{-1}$, and $100.2 \mathrm{~F} \cdot \mathrm{g}^{-1}$ with decay rates of $19.3 \%, 25.7 \%, 25.5 \%$, and $44.4 \%$, respectively, When the current density increases to $10.0 \mathrm{~A} \cdot \mathrm{g}^{-1}$. At a scan rate of $10 \mathrm{mV} \cdot \mathrm{s}^{-1}$, the CV curves of ZMPC-T-1:15 are showed in Figure 8(c). The curves all have similar rectangular shapes, which indicates that the composite porous carbon ZMPCT-1:15 has ideal bilayer capacitance at different carbonization temperatures, with ZMPC-800-1:15 having the largest integration area, showing its excellent capacitive performance. As showed in Figure 8(d), resistance values of ZMPC-T-1:15 are $1.96,1.72,1.55$, and $2.20 \Omega$, respectively, when the carbonization temperature are $600^{\circ} \mathrm{C}, 700^{\circ} \mathrm{C}, 800^{\circ} \mathrm{C}$, and $900^{\circ} \mathrm{C}$, respectively. The porous carbon ZMPC-800-1:15 composite carbon material shows the lowest resistance value, which corresponds with its largest specific surface area and electrochemical properties.

\subsubsection{Electrochemical Properties of Composite Porous Carbon ZMPC-800-1:15}

Figure 9 shows the electrochemical tests of the composite porous carbon ZMPC800-1:15. When current densities increase from $1.0 \mathrm{~A} \cdot \mathrm{g}^{-1}$ to $10.0 \mathrm{~A} \cdot \mathrm{g}^{-1}$, the constant current charge/discharge curves of the composite carbon material ZMPC-8001:15 are showed in Figure 9(a). At different current densities, the GCD curves show a symmetrical triangular shape, which indicates that the material has good electrochemical properties of the bilayer. Figure 9(b) shows that when the current density gradually increases from $1.0 \mathrm{~A} \cdot \mathrm{g}^{-1}$ to $10.0 \mathrm{~A} \cdot \mathrm{g}^{-1}$, the specific capacitance shows a smooth decaying trend, which is mostly attributable to the short reaction time that became shorter between the electrolyte ions and the carbon material at high current densities, the microporous charge of the carbon material is not filled effectively, and the microporous utilization rate of the carbon material is reduced. At different scan rates, Figure 9(c) shows the CV curves of ZMPC-800-1:15 are showed in Figure 9(c). With the scan rate increasing from 10 to $100 \mathrm{mV} \cdot \mathrm{s}^{-1}$, the shape of the $\mathrm{CV}$ curves remains similar to a rectangle. As showed in Figure 9(d), at a current density of $1.0 \mathrm{~A} \cdot \mathrm{g}^{-1}, \mathrm{ZMPC}-800-1: 15$ has a very small attenuation of specific capacitance and the capacitance retention rate is $96.41 \%$ after 5000 cycles. The result of experience indicates the material has excellent stable cycling stability, which provides an experimental basis for its ultralong cycling stability. 


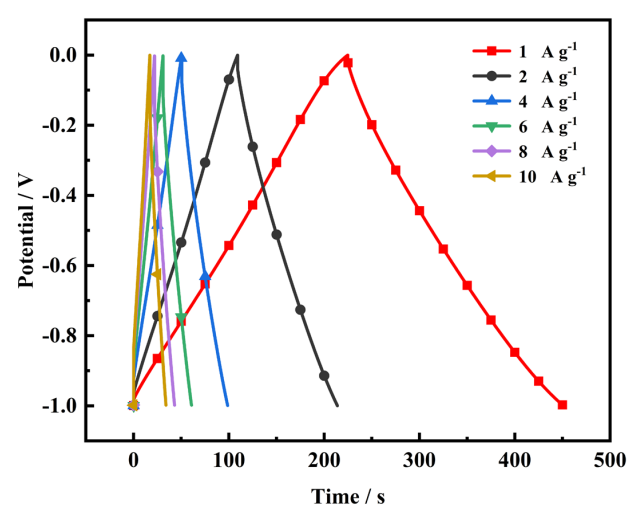

(a)

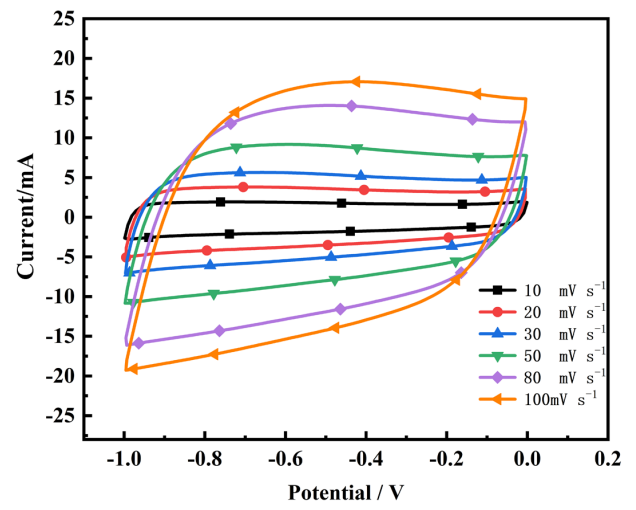

(c)

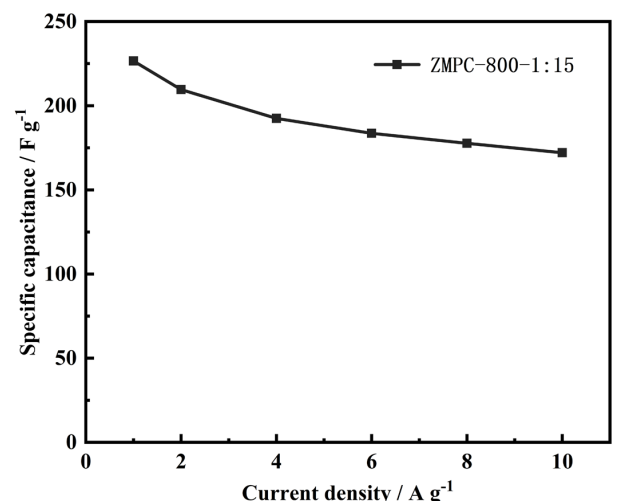

(b)

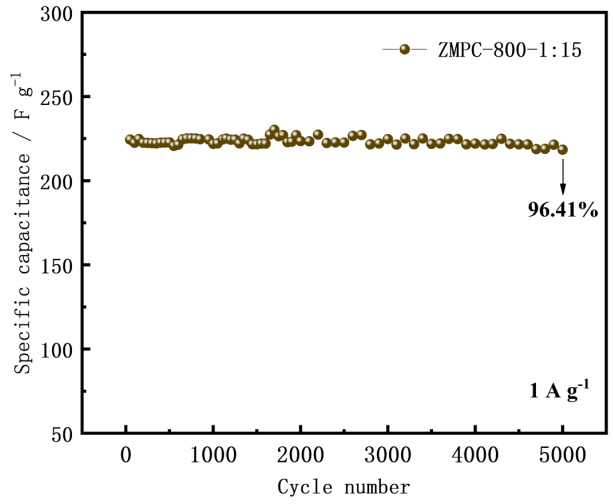

(d)

Figure 9. Electrochemical test of composite porous carbon ZMPC-800-1:15: (a) GCD curves at different current densities; (b) specific capacitance based on GCD curves; (c) CV curves at different scan rates; (d) cycling performance at a current density of $1.0 \mathrm{~A} \cdot \mathrm{g}^{-1}$.

\section{Conclusions}

1) The metal-organic framework material $Z M$ was prepared by the solution method, a mixture of $\mathrm{ZM}, \mathrm{ZM}$ and potassium citrate was used as carbon precursors, and the porous carbon ZMC-T and composite porous carbon ZMPC-T-A were prepared by the direct carbonization method at temperatures in the $600^{\circ} \mathrm{C}$ $900^{\circ} \mathrm{C}$ range. The maximum specific surface area of ZMPC-800-1:15 was 2014.97 $\mathrm{m}^{2} \cdot \mathrm{g}^{-1}$, and the material was graphitized amorphous. The pore size was mainly mesoporous and contained micropores and macropores.

2) At the current density from $1.0 \mathrm{~A} \cdot \mathrm{g}^{-1}$, the specific capacitance of MOF-derived porous carbons ZMC-800 and ZMPC-800-1:15 was 121.3 and $226.6 \mathrm{~F} \cdot \mathrm{g}^{-1}$, respectively, with a substantial increase of $86.8 \%$. With the current density increasing from 1.0 to $10.0 \mathrm{~A} \cdot \mathrm{g}^{-1}$, the decay rate of the specific capacitance was $25.5 \%$; the retention rate of capacitance remained $96.41 \%$ after 5000 cycles of constant current charging and discharging, and the composite porous carbon ZMPC-800-1:15 shown excellent electrochemical energy storage and a stable multiplication rate and cycling performance. This result of experience provides date support for preparing composite pour carbon material with a high specific surface area. 


\section{Acknowledgements}

This work is partially supported by the fund of National Nature Science Foundation of China (Nos. 21667017 and 21666018) and Gansu Province University Fundamental Research Funds (No. 056002).

\section{Conflicts of Interest}

The authors declare no conflicts of interest regarding the publication of this paper.

\section{References}

[1] Najib, S. and Erdem, E. (2019) Current Progress Achieved in Novel Materials for Supercapacitor Electrodes: Mini Review. Nanoscale Advances, 1, 2817-2827. https://doi.org/10.1039/C9NA00345B

[2] Du, W., Bai, Y.-L., Xu, J., Zhao, H., Zhang, L., Li, X. and Zhang, J. (2018) Advanced Metal-Organic Frameworks (MOFs) and Their Derived Electrode Materials for Supercapacitors. Journal of Power Sources, 402, 281-295. https://doi.org/10.1016/j.jpowsour.2018.09.023

[3] Jiang, L., Wang, J., Mao, X., Xu, X., Zhang, B., Yang, J., Wang, Y., Zhu, J. and Hou, S. (2017) High Rate Performance Carbon Nano-Cages with Oxygen-Containing Functional Groups as Supercapacitor Electrode Materials. Carbon, 111, 207-214. https://doi.org/10.1016/j.carbon.2016.09.081

[4] Pandolfo, A.G. and Hollenkamp, A.F. (2006) Carbon Properties and Their Role in Supercapacitors. Journal of Power Sources, 157, 11-27. https://doi.org/10.1016/j.jpowsour.2006.02.065

[5] Chen, L.-F., Zhang, X.-D., Liang, H.-W., Kong, M., Guan, Q.-F., Chen, P., Wu, Z.-Y. and Yu, S.-H. (2012) Synthesis of Nitrogen-Doped Porous Carbon Nanofibers as an Efficient Electrode Material for Supercapacitors. ACS Nano, 6, 7092-7102. https://doi.org/10.1021/nn302147s

[6] Endo, M., Takeda, T., Kim, Y.J., Koshiba, K. and Ishii, K. (2001) High Power Electric Double Layer Capacitor (EDLC’s); From Operating Principle to Pore Size Control in Advanced Activated Carbons. Carbon Letters, 1, 117-128.

[7] Yue, M.-L., Jiang, Y.-F., Zhang, L., Yu, C.-Y., Zou, K.-Y. and Li, Z.-X. (2017) Solvent-Induced Cadmium(II) Metal-Organic Frameworks with Adjustable Guest-Evacuated Porosity: Application in the Controllable Assembly of MOF-Derived Porous Carbon Materials for Supercapacitors. Chemistry, 23, 15680-15693.

https://doi.org/10.1002/chem.201702694

[8] Gangu, K.K., Maddila, S., Mukkamala, S.B. and Jonnalagadda, S.B. (2019) Characteristics of MOF, MWCNT and Graphene Containing Materials for Hydrogen Storage: A Review. Journal of Energy Chemistry, 30, 132-144. https://doi.org/10.1016/j.jechem.2018.04.012

[9] Yang, J., Zheng, C., Xiong, P., Li, Y. and Wei, M. (2014) Zn-doped Ni-MOF Material with a High Supercapacitive Performance. Journal of Materials Chemistry A, 2, 19005-19010. https://doi.org/10.1039/C4TA04346D

[10] O’Keeffe, M. and Yaghi, O.M. (2011) Deconstructing the Crystal Structures of Metal-Organic Frameworks and Related Materials into Their Underlying Nets. Chemical reviews, 112, 675-702. https://doi.org/10.1021/cr200205j

[11] Hong, S., Kim, Y., Kim, Y., Suh, K., Yoon, M. and Kim, K. (2021) Hierarchical Porous Carbon Materials Prepared by Direct Carbonization of Metal-Organic Frame- 
works as an Electrode Material for Supercapacitors. Bulletin of the Korean Chemical Society, 42, 309-314. https://doi.org/10.1002/bkcs.12145

[12] Zhong, S., Kitta, M. and Xu, Q. (2019) Hierarchically Porous Carbons Derived from Metal-Organic Framework/Chitosan Composites for High-Performance Supercapacitors. Chemistry-An Asian Journal, 14, 3583-3589.

https://doi.org/10.1002/asia.201900318

[13] Jiang, M., Ca, O.X., Liu, P., Zhang, T. and Zhang, J. (2016) ZIF-8@Polyvinylpyrrolidone Nanocomposites Based N-Doped Porous Carbon for Highly Efficient Oxygen Reduction Reaction in Alkaline Solution. Journal of the Electrochemical Society, 163, H459-H464. https://doi.org/10.1149/2.1231606jes

[14] Zhang, C., Wang, P., Li, S., Zhang, J. and Luo, H. (2021) Preparation and Electrochemical Properties of MOF-Derived Nitrogen Self-Doped Porous Carbon. Journal of the Iranian Chemical Society. https://doi.org/10.1007/s13738-021-02257-1

[15] Wang, C., Yin, H., Tian, P., Sun, X., Pan, X., Chen, K., Chen, W.-J., Wu, Q.-H. and Luo, S. (2020) Remarkable Adsorption Performance of MOF-199 Derived Porous Carbons for Benzene Vapor. Environmental Research, 184, Article ID: 109323. https://doi.org/10.1016/j.envres.2020.109323

[16] Cendrowski, K., Skumial, P., Spera, P. and Mijowska, E. (2016) Thermally Induced Formation of Zinc Oxide Nanostructures with Tailoring Morphology during Metal Organic Framework (MOF-5) Carbonization Process. Materials \& Design, 110 , 740-748. https://doi.org/10.1016/j.matdes.2016.08.043

[17] Pal, S., Sivasankar, K., Thiruppathi, M. and Lin, C.H. (2020) Carbonization and Preparation of Nitrogen-Doped Porous Carbon Materials from Zn-MOF and Its Applications. Materials, 2, Article No. 264. https://doi.org/10.3390/ma13020264

[18] Zhang, Y., Nie, T., Wang, Z., Liu, J. and Cen, K. (2016) Splitting of $\mathrm{CO}_{2}$ via the Heterogeneous Oxidation of Zinc Powder in Thermochemical Cycles. Industrial \& Engineering Chemistry Research, 55, 534-542. https://doi.org/10.1021/acs.iecr.5b02407

[19] Krishnamoorthy, K., Veerapandian, M., Yun, K. and Kim, S.J. (2013) The Chemical and Structural Analysis of Graphene Oxide with Different Degrees of Oxidation. Carbon, 53, 38-49. https://doi.org/10.1016/j.carbon.2012.10.013

[20] Vilcinskas, K., Mulder, F.M., Picken, S.J. and Koper, G. (2017) In Situ X-Ray Diffraction Studies of Graphite Oxidation Reaction Indicating Different Exfoliation Mechanism than EX Site Studies. arXiv Preprint, arXiv: 1701.06493.

[21] Sharma, S.K. and Exarhos, G.J. (1997) Raman Spectroscopic Investigation of ZnO and Doped ZnO Films, Nanoparticles and Bulk Material at Ambient and High Pressures. Solid State Phenomena, 55, 32-37. https://doi.org/10.4028/www.scientific.net/SSP.55.32

[22] Wang, M.-L. and Bian, W.-F. (2020) The Relationship between the Mechanical Properties and Microstructures of Carbon Fibers. New Carbon Materials, 35, 42-49. https://doi.org/10.1016/S1872-5805(20)60474-7

[23] Mohan, A.N., Manoj, B. and Ramya, A.V. (2016) Probing the Nature of Defects of Graphene Like Nano-Carbon from Amorphous Materials by Raman Spectroscopy. Asian Journal of Chemistry, 28, 1501-1504. https://doi.org/10.14233/ajchem.2016.19739

[24] Chaikittisilp, W., Ariga, K. and Yamauchi, Y. (2013) A New Family of Carbon Materials: Synthesis of MOF-Derived Nanoporous Carbons and Their Promising Applications. Journal of Materials Chemistry A, 1, 14-19. https://doi.org/10.1039/C2TA00278G

[25] Thommes, M. (2016) Physisorption of Gases, with Special Reference to the Evaluation of Surface Area and Pore Size Distribution (IUPAC Technical Report). Pure 
and Applied Chemistry, 87, 25-25. https://doi.org/10.1515/ci-2016-0119

[26] Sevilla, M. and Fuertes, A.B. (2014) Direct Synthesis of Highly Porous Interconnected Carbon Nanosheets and Their Application as High-Performance Supercapacitors. ACS Nano, 8, 5069-5078. https://doi.org/10.1021/nn501124h

[27] Sevilla, M. and Fuertes, A.B. (2013) A General and Facile Synthesis Strategy towards Highly Porous Carbons: Carbonization of Organic Salts. Journal of Materials Chemistry $A, 1,13738-13741$. https://doi.org/10.1039/c3ta13149a

[28] Ferrero, G.A., Sevilla, M. and Fuertes, A.B. (2015) Mesoporous Carbons Synthesized by Direct Carbonization of Citrate Salts for Use as High-Performance Capacitors. Carbon, 88, 239-251. https://doi.org/10.1016/j.carbon.2015.03.014

[29] Zahoor, A., Christy, M., Hwang, Y.J., Lim, Y.R., Kim, P. and Nahm, K.S. (2014) Improved Electrocatalytic Activity of Carbon Materials by Nitrogen Doping. Applied Catalysis B: Environmental, 147, 633-641.

https://doi.org/10.1016/j.apcatb.2013.09.043

[30] Estrade-Szwarckopf, H. (2004) XPS Photoemission in Carbonaceous Materials: A "Defect" Peak beside the Graphitic Asymmetric Peak. Carbon, 42, 1713-1721. https://doi.org/10.1016/j.carbon.2004.03.005

[31] Zielke, U., Hüttinger, K. and Hoffman, W.P. (1996) Surface-Oxidized Carbon Fibers: I. Surface Structure and Chemistry. Carbon, 34, 983-998. https://doi.org/10.1016/0008-6223(96)00032-2

[32] Chen, X.N., Wang, X.H. and Fang, D. (2020) A Review on C1s XPS-Spectra for Some Kinds of Carbon Materials. Fullerenes Nanotubes and Carbon Nanostructures, 28, 1048-1058. https://doi.org/10.1080/1536383X.2020.1794851

[33] Tian, Q., Wang, X.X., Xu, X.Y., Zhang, M., Wang, L.Y., Zhao, X.X., An, Z.L., Yao, H.D. and Gao, J.P. (2018) A Novel Porous Carbon Material Made from Wild Rice Stem and Its Application in Supercapacitors. Materials Chemistry and Physics, 213, 267-276. https://doi.org/10.1016/j.matchemphys.2018.04.026 